\title{
PUQ: a code for non-intrusive uncertainty propagation in computer simulations
}

\author{
Martin Hunt $^{\mathrm{a}}$, Benjamin Haley ${ }^{\mathrm{a}}$, Michael McLennan ${ }^{\mathrm{a}}$, Marisol Koslowski ${ }^{\mathrm{b}}$, \\ Jayathi Murthy ${ }^{\mathrm{d}}$, Alejandro Strachan ${ }^{1, *}$ \\ ${ }^{a}$ Research Computing, Purdue University, West Lafayette, Indiana 47906, USA \\ ${ }^{b}$ School of Mechanical Engineering, Purdue University, West Lafayette, Indiana 47906, \\ USA \\ ${ }^{c}$ Department of Mechanical Engineering, The University of Texas at Austin, Austin, \\ Texas 78712, USA \\ ${ }^{d}$ School of Materials Engineering and Birck Nanotechnology Center, Purdue University, \\ West Lafayette, Indiana 47906, USA
}

\begin{abstract}
We present a software package for the non-intrusive propagation of uncertainties in input parameters though computer simulation codes or mathematical models and associated analysis; we demonstrate its use to drive micromechanical simulations using a phase field approach to dislocation dynamics. The PRISM uncertainty quantification framework (PUQ) offers several methods to sample the distribution of input variables and to obtain surrogate models (or response functions) that relate the uncertain inputs with the quantities of interest (QoIs); the surrogate models are ultimately used to propagate uncertainties. PUQ requires minimal changes in the simulation code, just those required to annotate the QoI(s) for its analysis. Collocation methods include Monte Carlo, Latin Hypercube and Smolyak sparse grids and surrogate models can be obtained in terms of radial basis functions and via
\end{abstract}

\footnotetext{
*Corresponding author: strachan@purdue.edu. Phone: +1 (765) 490-5628. FAX: +1 (765) 494-1204
}

Preprint submitted to Computer Physics Communications

April 7, 2015 
generalized polynomial chaos. PUQ uses the method of elementary effects for sensitivity analysis in Smolyak runs. The code is available for download and also available for cloud computing in nanoHUB. PUQ orchestrates runs of the nanoPLASTICITY tool at nanoHUB where users can propagate uncertainties in dislocation dynamics simulations using simply a web browser, without downloading or installing any software.

Keywords: Uncertainty Quantification, Surrogate Model, Sensitivity

Analysis

\section{Program summary}

Program Title: PUQ

Journal Reference:

Catalogue identifier:

Licensing provisions: MIT license

Programming language: Python, $C$

Operating system: Linux, Mac OSX

Classification: 4.11, 4.12, 4.13

External routines/libraries: SciPy, Matplotlib, h5py

Nature of problem: Uncertainty propagation and creation of response surfaces.

Solution method: Generalized Polynomial Chaos ( $g P C$ ) using Smolyak sparse grids. 


\section{Introduction}

Computer simulations are playing an increasingly central role in engineering and science applications. Examples include the use of computational fluid dynamics to reduce the number of experiments in airframe design [1], virtual aluminum casting [2] and the US nuclear weapons program [3]. Models and their implementation in computer codes can only be used with confidence for such applications if the associated uncertainties have been quantified. Uncertainties in computer simulations have disparate origins but they can grouped into a few categories. i) Uncertainties associated with the numerical implementation of the model, such as those associated with finite discretization of space (mesh) or time. These are typically called verification uncertainties and can be reduced at a computational cost. ii) Uncertainties in the prediction due to model inappropriateness that stem from lack of knowledge. These uncertainties can only be quantified against appropriately designed and conducted experiments. iii) Uncertainties in the prediction due to uncertainties or variability in model's input parameters. This last type of uncertainty is the focus of this paper. Uncertain input parameters can be due to natural variability (aleatoric uncertainties) or lack of knowledge (epistemic) and the PRISM Uncertainty Quantification (PUQ) code enables users to propagate such uncertainties through a model with minimal modifications to the simulation code that implements it.

The brute-force propagation of uncertainties in input parameters through computationally intensive simulation codes is undesirable and often prohibitive. In many of these cases the use of surrogate models or response surfaces created from a relative small evaluations of the code represents a 
powerful tool for uncertainty propagation (UP) and this is the approach taken by PUQ. The challenge then lies in the choice of functional form for the surrogate model, the optimal selection of collocation points to perform the simulations given the distribution of input variables and the parameterization of the surrogate based on the data collected.

This paper introduces the methods implemented in PUQ and demonstrates its use to create surrogate models to represent a function often used in the UQ literature and for the propagation on uncertainties in input parameters through a micromechanical model of nano crystalline plasticity of interest in materials science. PUQ allows for the propagation of uncertainties in input parameters with minimal changes in the simulation code, using several collocation techniques including Monte Carlo, Latin Hypercube, and sparse grids and to obtain surrogate models based on radial basis sets and polynomial chaos. PUQ was designed with ease of use and non-intrusiveness in mind. We also introduce nanoPLASTICITY, [4] a tool for online simulations in nanoHUB [5, 6] that uses PUQ to drive a phase field simulator of dislocation dynamics. nanoPLASTICITY users can specify inputs as single values or probability distributions and the tool automatically propagates uncertainties and displays results in a graphical and fully interactive manner. nanoHUB enables free simulations in the cloud via a standard web-browser, users do not need to download and install any software nor to provide compute cycles. 


\section{Uncertainty propagation and sensitivity}

Uncertainty propagation involves finding the distribution of outputs given known distributions of inputs to a simulation code. Assume the quantity of interest, $q$ depends on input parameter $p$ :

$$
q=Q(p)
$$

where both $q$ and $p$ can, in general be vectors or even functions [7]. If the input is uncertain and represented by random variable $P$ the output will also be a random variable, denoted $Q$. We would like to obtain the distribution that characterizes $Q$ given the distribution of $P$. A direct Monte Carlo approach would involve drawing input parameters $\left\{p_{i}\right\}$ from the known distributions and evaluating the code to sample the output distribution: $q_{i}=$ $Q\left(p_{i}\right)$. As mentioned above, this is often prohibitively expensive and PUQ uses a surrogate model $\hat{Q}$ that is computationally inexpensive to propagate uncertainties. This is a powerful method if the number of evaluations of the actual simulation required to develop the surrogate is small compared to the number of evaluations required for UP. The disadvantage is clearly that the resulting output PDF will be an approximation that depends on the fidelity of $\hat{Q}$ over the range of likely input parameters.

This section discusses the methods used for sampling, extraction of surrogate models and sensitivity analysis available in PUQ. We also compare the relative performance of the various methods. Sampling can be Monte Carlo sampling, Latin hypercube (LHC) and Smolyak sparse grids. Surrogate models can be developed in terms of radial basis functions or via generalized polynomial chaos (gPC). PUQ provides sensitivity analysis using 
the elementary effects method.

\subsection{Input distributions}

The uncertain input variables in PUQ can be given as uniform, normal, exponential, Rayleigh, and Weibull PDFs. Additional distributions can be easily added. PUQ also supports custom PDFs, which can be imported from previous PUQ runs, built from collected data, or input as a 2D array of histogram values and heights.

PDFs built from collected data use Gaussian kernel density estimation. In this case, the default bandwidth is computed using Scott's Rule [8], but any bandwidth can be used.

\subsection{UQ methods}

\subsubsection{Monte Carlo}

The most direct UQ method available in PUQ is Monte Carlo (MC). Samples are drawn directly from the parameter distributions. Monte Carlo is the simplest sampling method and the easiest to extend; each sample is independent of the others so adding additional samples can be done at any time. However, $\mathrm{MC}$ is very slow, requiring an excessive number of runs to get consistent results; thus it is not recommended except when surrogates are not possible or when the simulations are computationally very inexpensive. The convergence rate for the mean is $O(1 / \sqrt{N})$

\subsubsection{Latin Hypercube}

Latin Hypercube sampling [9] is generally an improvement over MC sampling. It is a stratified method that divides the input parameter ranges into 
equal probability intervals, then chooses one random value in each interval. This is done in each dimension, then the values are randomly combined. By doing this, it avoids the clustering and uneven sampling issues that slow down the convergence of MC.

For $N$ random variables and $M$ intervals, the total number of runs will be $M . M$ is independent of $N$, however in reality, it must be increased as $N$ increases in order to have sufficient coverage of the input space. Convergence is faster than $\mathrm{MC}$, but achieving convergences becomes onerous as the number of input parameters increases.

\subsubsection{Generalized polynomial chaos}

The polynomial chaos expansion method is a powerful tool to relate distributions of input parameters to distributions of outputs. [10] The term chaos originates from statistical mechanics [11] where integrals over time can be replaced by integrals over phase space (equivalence between time and ensemble averaging) for ergodic systems; i.e. distributions over one variable can be mapped to distributions over different ones. The term polynomial indicates that a polynomial will be used to relate input to output PDFs. The basic idea is to express the surrogate $\hat{Q}$ using a basis set of orthogonal polynomials.

$$
\hat{Q}(z)=\sum_{k=0}^{N} u_{k} \Phi_{k}(z)
$$

where $\Phi_{k}(z)$ is the $k$ th interpolating polynomial and $u_{k}$ is the corresponding coefficient.

Once we choose the interpolating polynomials, our problem is then reduced to finding the vector of coefficients, $u$. Examining orthogonality prop- 
erties of orthogonal polynomials, we can show that

$$
u_{k}=\frac{\left\langle Q, \Phi_{k}\right\rangle}{\left\langle\Phi_{k}^{2}\right\rangle}=\frac{\int Q(z) \Phi_{k}(z) \rho(z)}{\left\langle\Phi_{k}^{2}\right\rangle}
$$

\subsubsection{Choosing a polynomial basis}

Xiu and Karniadakis[10] observed that using the Hermite polynomials with gPC resulted in optimal convergence only when the input distributions were Gaussian. Other input distributions required different polynomials for optimal convergence. In PUQ, we wanted to support input parameters with a mix of distributions, as well as custom distributions. To achieve this, we initially treat all input PDFs as uniform and use Legendre polynomials.

To evaluate the integral in the numerator of Eq. 3, we use sparse grid Smolyak cubature with Clenshaw-Curtis points. This greatly reduces the number of collocation points while preserving a high level of accuracy [12].

Using cubature to approximate the integral, and with a constant for the input distribution $\rho$, Eq. 3 now becomes something we can use to efficiently compute the coefficients,

$$
\hat{u_{k}}=\sum_{i=0}^{N_{c}} Q\left(x_{i}\right) \Phi_{k}\left(x_{i}\right) w_{i}
$$

where $N_{c}$ is the number of collocation points, $x_{i}$ is the $i$ th collocation point, $Q\left(x_{i}\right)$ is the result of the simulation at that point, $\Phi_{k}\left(x_{i}\right)$ is the $k$ th polynomial evaluated at $x_{i}$, and $w_{i}$ is the collocation weight.

This is a response surface approximation to $Q(z)$. Finally, to compute the output $\operatorname{PDF}(\mathrm{s})$, we sample all the input parameters using their proper PDFs and evaluate the response. This is done by evaluating the response surface with a large number of samples chosen with LHS. The output values 
are then used to compute the mean, variance, and the output $\mathrm{PDF}(\mathrm{s})$.

\subsection{Other surrogate models}

Polynomial response surfaces built from gPC are not the only surrogate models PUQ supports. Radial basis functions can also be used to build a response surface from any sample points; MC, LHS, or sparse grids. Not all responses can be fit to a polynomial, so this provides an alternative for those cases. PUQ supports the radial basis functions in SciPy [13]: multiquadric, inverse, Gaussian, linear, cubic, quintic, and thin plate.

\subsection{Sensitivity}

Sensitivity analysis is performed using the elementary effects method developed by Morris[14] and Campolongo and Saltelli[15]. This is a one-ata-time method that measures the change in output when one input variable is changed while all others are held constant. This is done at multiple points and the results averaged, giving us the sensitivity measure $\mu *$. PUQ uses sample points chosen from the same sparse grid it used to build the response surface. More details on how this is done can be found at http://c-primed.github.io/puq/ug/sensitivity.html.

\section{Convergence studies}

To illustrate the diferences between Monte Carlo sampling, Latin Hypercube sampling, and gPC with the Smolyak sparse grid, we will use the methods to estimate the mean of two different functions. For MC and LHS, the mean of the function is simply the mean of all the sample points. Because of the randomness in the methods, each method was run 1000 times. The 


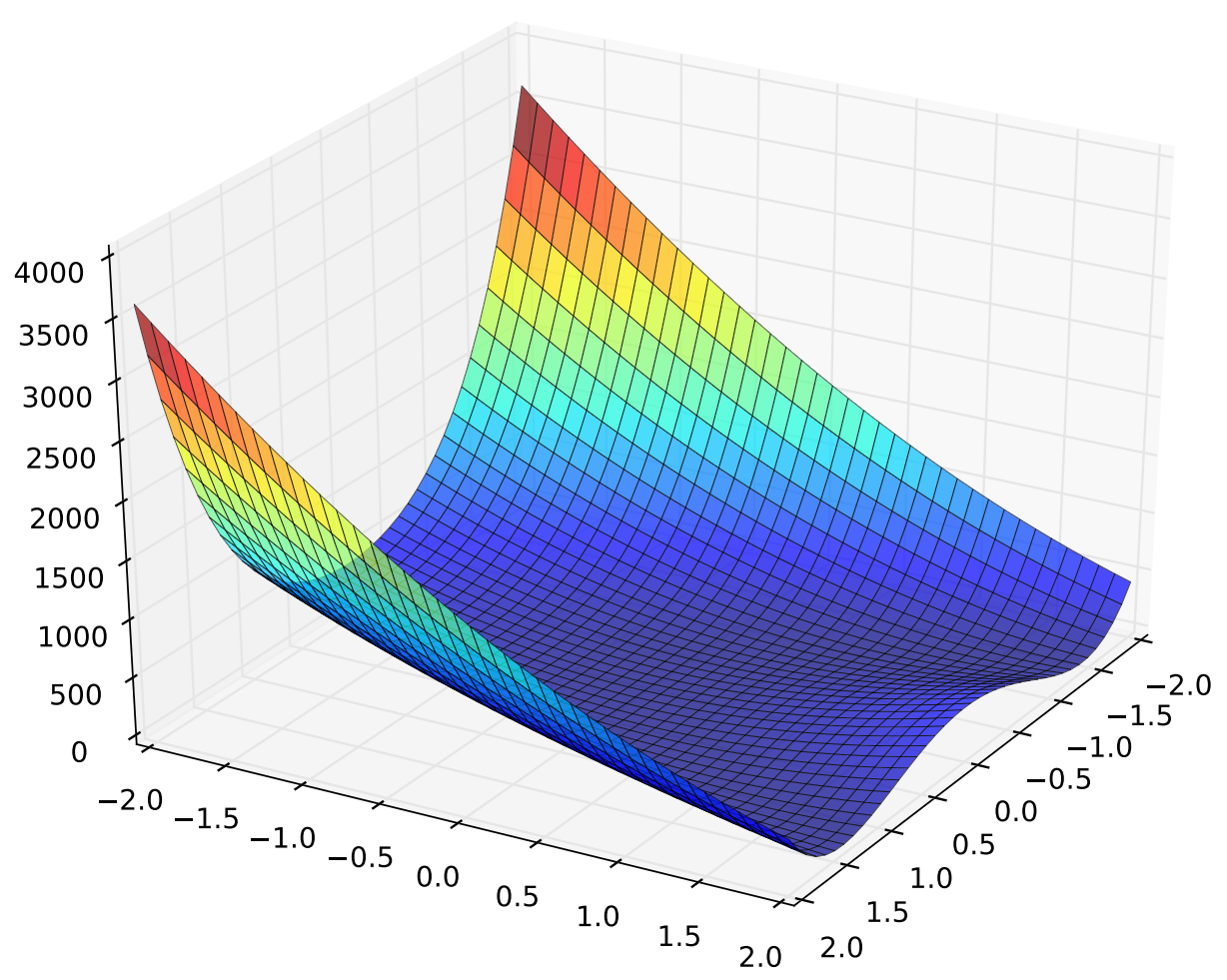

Figure 1: Rosenbrock function $f(x, y)=100\left(y-x^{2}\right)^{2}+(1-x)^{2}$

convergence plot shows the maximum error in all the runs. For the Smolyak gPC method, the sample points are not chosen at random, so only one run was performed.

\subsection{Test functions}

The two-dimensional Rosenbrock function (Fig. 1) is sampled independently in both dimensions on the interval $[-2,2]$. It is a polynomial, so it should work well with gPC. Its mean in this region can be computed as

$$
\frac{1}{16} \int_{-2}^{2} \int_{-2}^{2} 100\left(y-x^{2}\right)^{2}+(1-x)^{2} d x d y=455 \frac{2}{3}
$$


The one-dimensional decay function, $e^{-x}$ is not a polynomial, so gPC will need a higher-order polynomial to approximate it. However, for this study, we are just interested in the mean on the interval $[-3,3]$ for a uniform distribution. Again we can easily compute this analytically,

$$
\frac{1}{6} \int_{-3}^{3} e^{-x} d x=-\frac{1}{6 e^{3}}+\frac{e^{3}}{6}=3.33929164247
$$

\subsection{Results}

Figures 2 and 3 plot the error percent in the estimate of the mean versus the number of sample points. In both cases we see LHS converging faster than MC, as expected. We also see a dramatic improvement on convergence with the Smolyak gPC method.

\section{PUQ examples}

PUQ was created with two goal in mind: i) making it easy to use with various simulations codes and, ii) to be easily integrated and extended.

\subsection{Implementation}

PUQ is implemented in Python and has a command-line interface, GUI, and scripting API. It does non-intrusive UQ on simulations. The simulation can be written in any language. PUQ only needs to know how to pass parameters to the simulation and read the QoI. Currently PUQ expects to be able to pass parameters on the command line. A future version will be able to make use of simple template files to make passing parameters simpler for simulations that require parameters to be in input files. More complicated situations require creation of a wrapper script which will take command line 
50

51

52

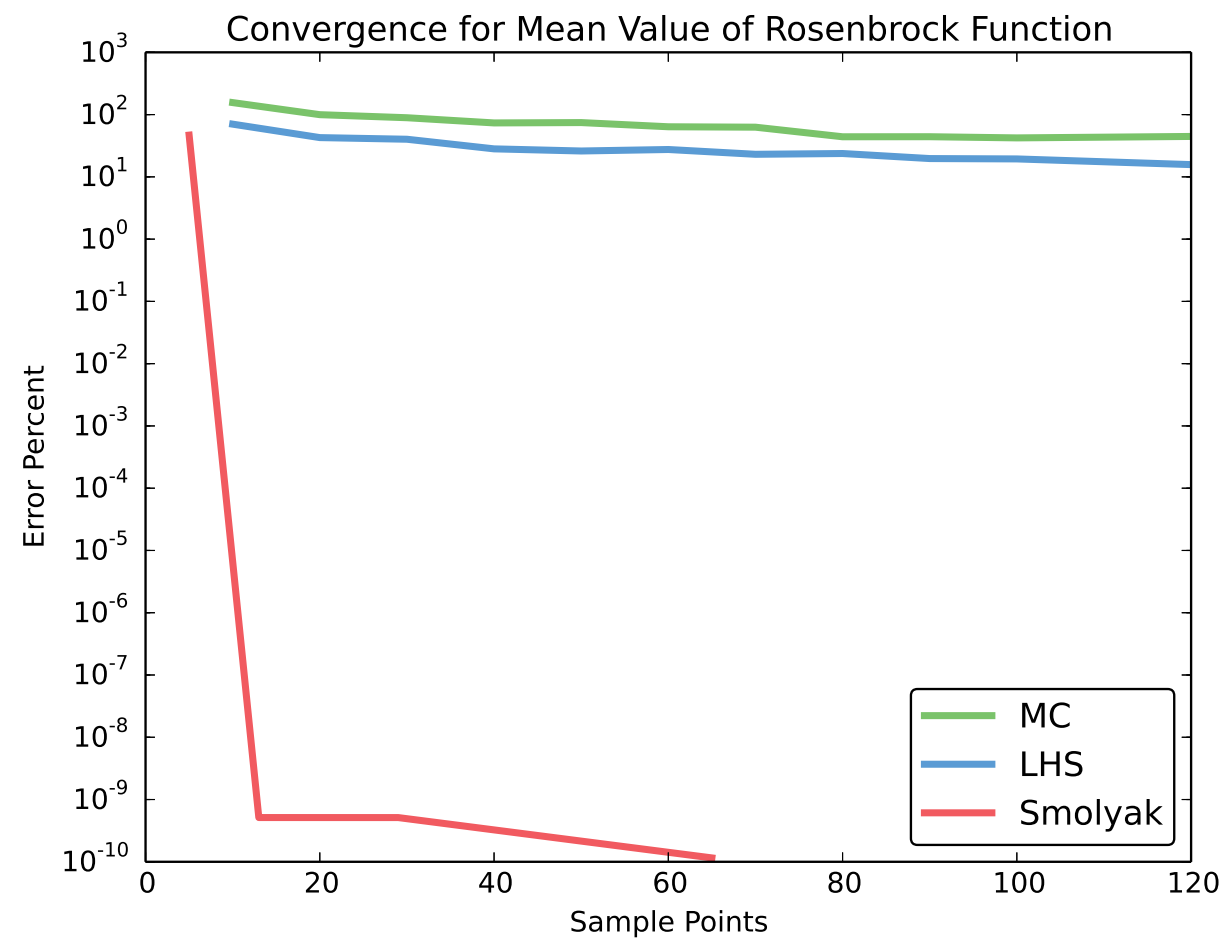

Figure 2: Convergence for Rosenbrock function 
50

51

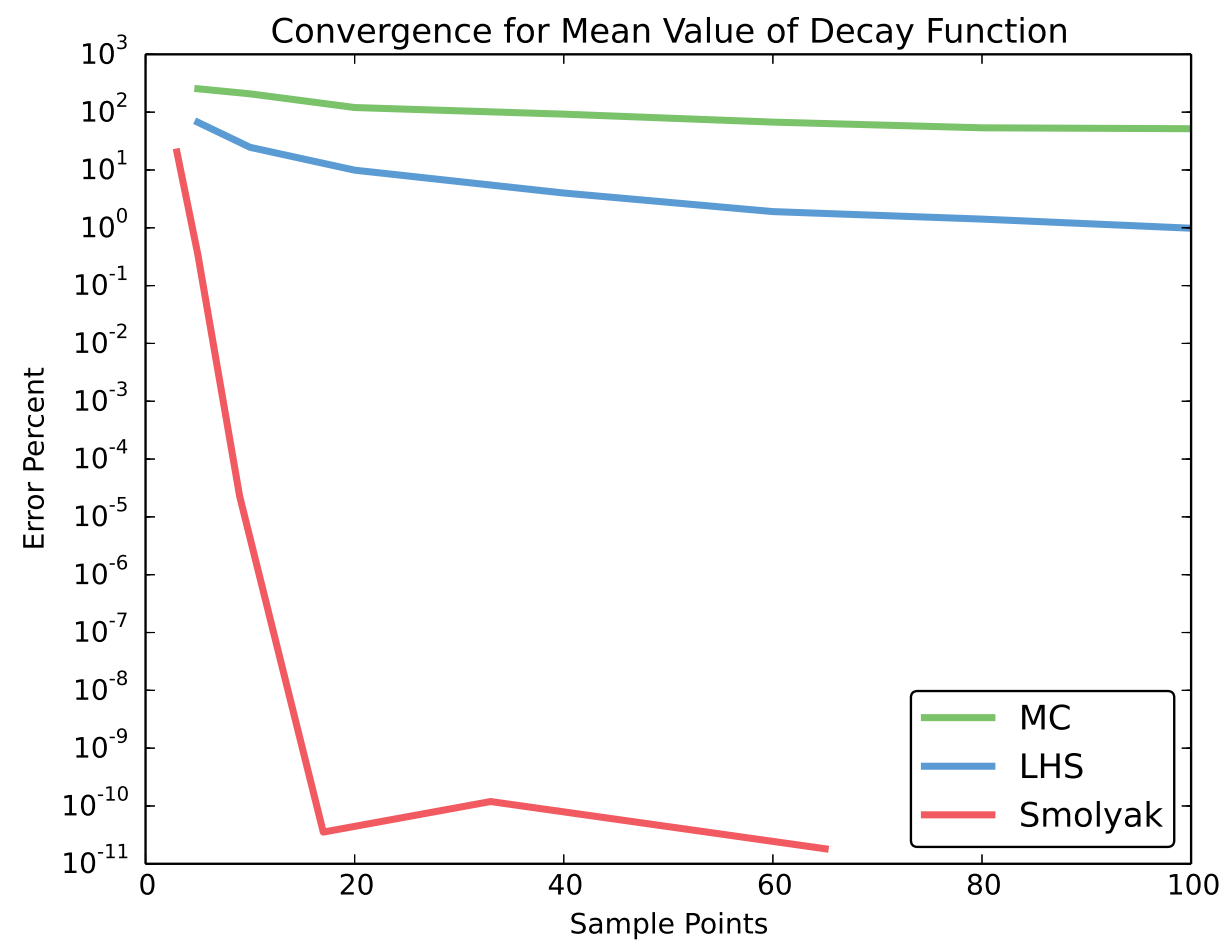

Figure 3: Convergence for decay function 


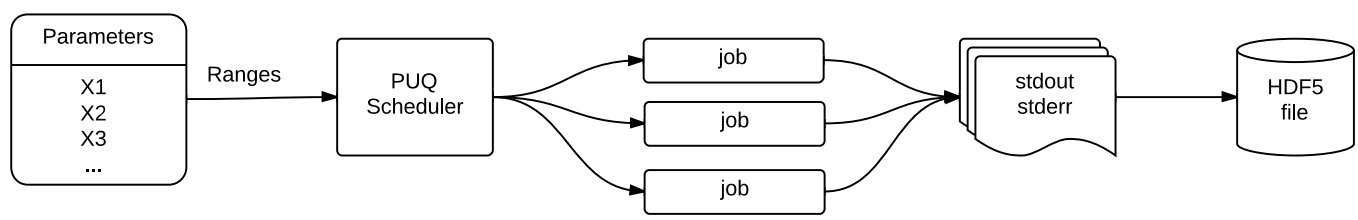

Figure 4: PUQ runs multiple jobs with different values for input parameters and collects the output into an HDF5 file.

parameters, run the simulation with them, and return the QoI. PUQ can handle multiple quantities of interest and will perform UQ and sensitivity analysis on each.

Input parameters must be continuous probability density functions (PDFs). Currently PUQ handles uniform, normal, exponential, Rayleigh, and Weibull PDFs. Additional distributions can be easily added. PUQ also supports custom PDFs, which are built from collected data using Gaussian kernel density estimation. PUQ can read saved PDFs from a database or URL, allowing simulations to easily use the output of previous simulations.

PUQ can run hundreds or thousands of jobs, each of which may create several files. It collects all this into one large HDF5 file then analyzes the collected data. This process may then be automatically repeated until some condition is reached. PUQ contains a GUI that allows users to open this file and see the raw data files, view and export the collected data, see the input PDFs, and visualize the sensitivity, response surfaces and output PDF(s).

\subsection{Uncertainty propagation via Monte Carlo sampling}

Monte Carlo is the simplest sampling method included in PUQ and is provided as a reference, but its use is discouraged due to its lower performance compared to other approaches. By default, when Monte Carlo sampling 


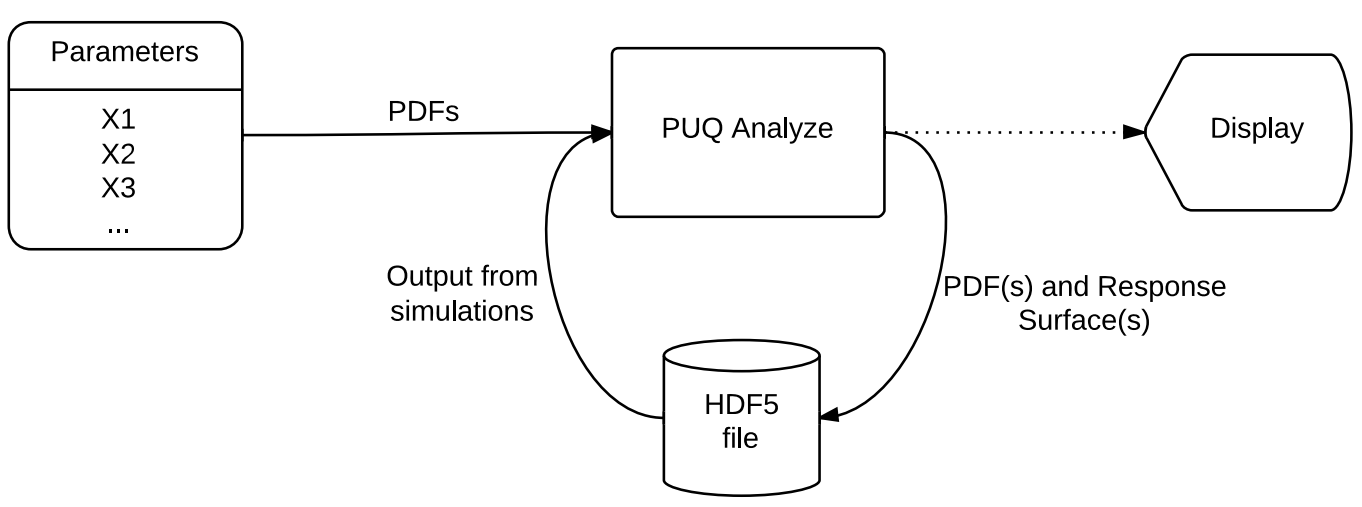

Figure 5: The PUQ analysis phase builds response surfaces and output PDFs.

is used a response surface approximation will be built using radial basis functions.

We will again use the Rosenbrock function as an example and demonstrate how to use PUQ to build a response surface and get an output PDF. The simulation code rosen_prog.py below takes two arguments and computes the Rosenbrock function. The result is returned using the dump_hdf5() function which tags the data in the output stream so it can be easily stripped out and stored in the HDF5 output file.

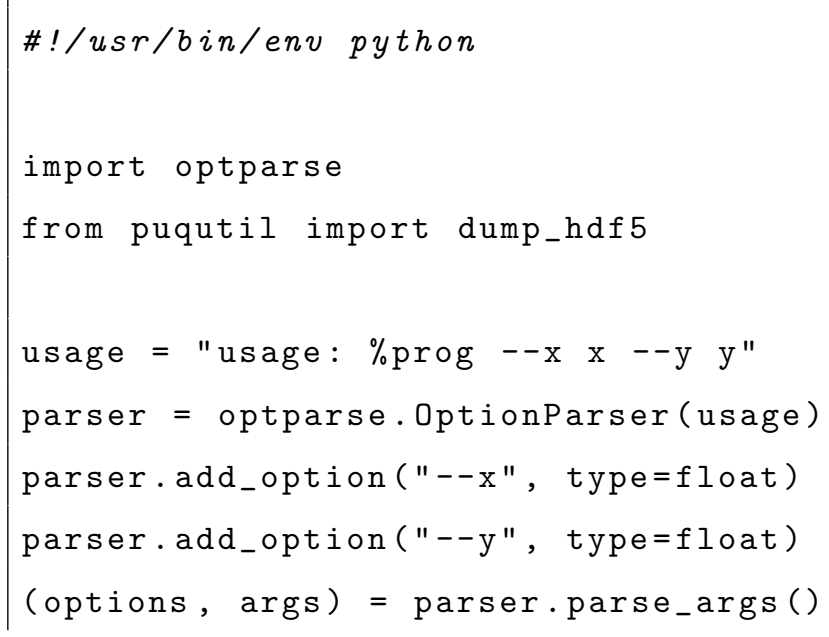


$x=$ options. $x$

$\mathrm{y}=$ options $\cdot \mathrm{y}$

$\mathrm{z}=100 *(\mathrm{y}-\mathrm{x} * * 2) * * 2+(1-\mathrm{x}) * * 2$

dump_hdf5('z', z)

After execution the program outputs the value of the Rosenbrock function as an HDF5 line.

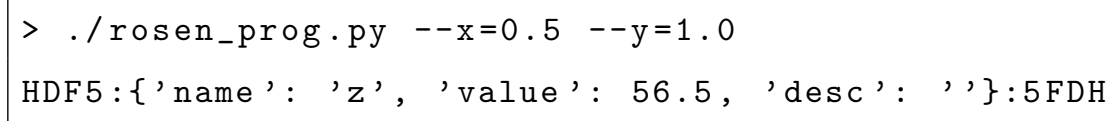

This illustrates the format that PUQ expects to find the QoI; the HDF5 line contains a name, numerical value, and a description. The values are, of course, used to compute the response surfaces and the name and descriptions to keep track of possible multiple QoIs.

A Python script is used to setup up and run PUQ; very little Python knowledge is required. Below is an example that calls the Rosenbrock program above, rosen_mc.py.

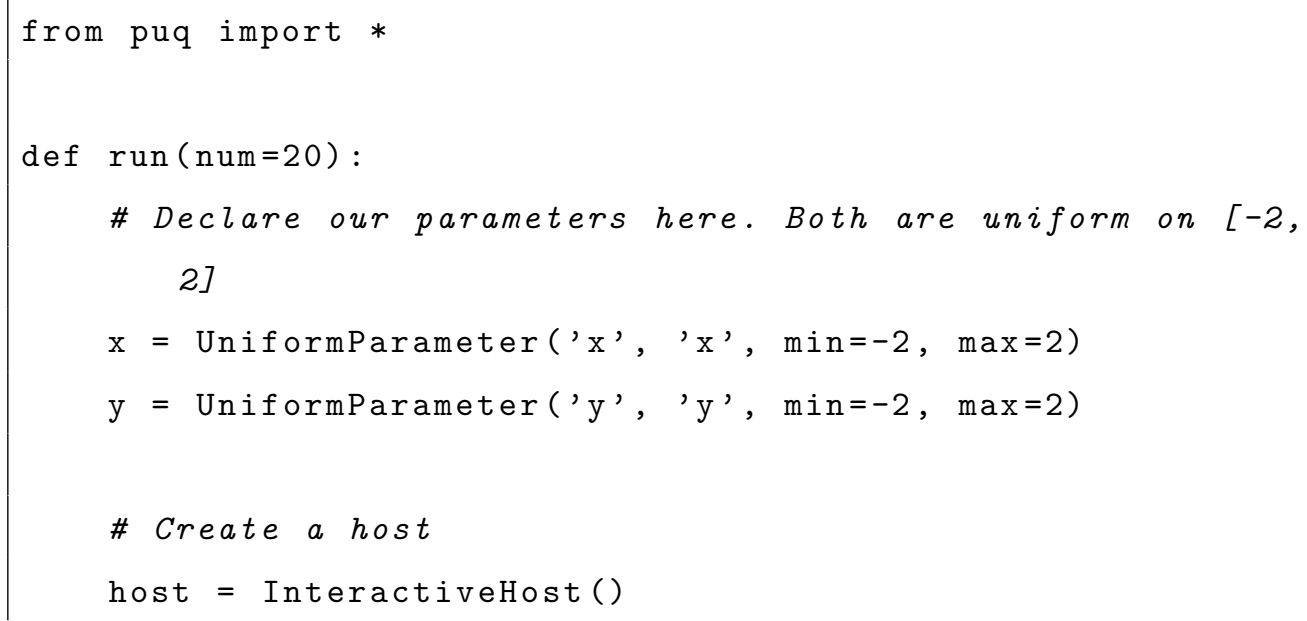


$\mathrm{uq}=\operatorname{MonteCarlo}([\mathrm{x}, \mathrm{y}]$, num $=\mathrm{num})$

\# Our test program

prog = TestProgram(',/rosen_prog.py', desc='Rosenbrock Function (python)')

return Sweep (uq, host, prog)

PUQ can then be invoked with the output file name, script and additional inputs as command line arguments.

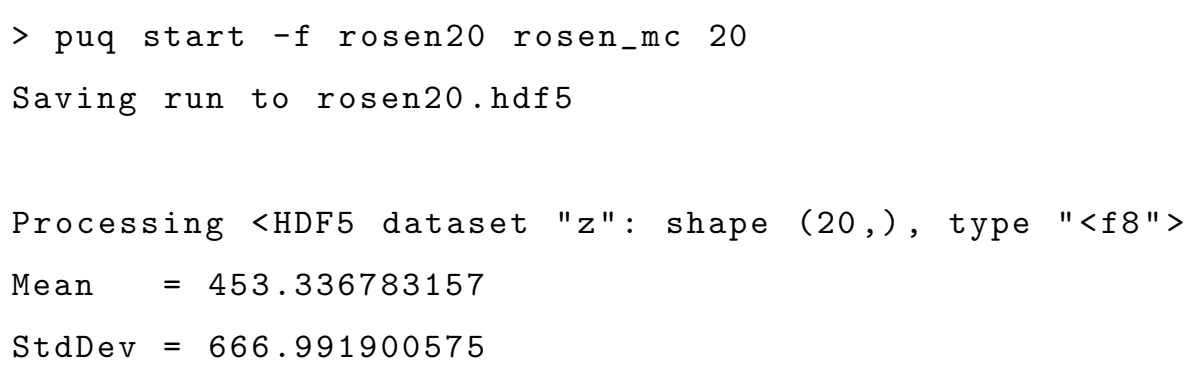

The output can be analyzed using PUQ's GUI:

$>$ puq analyze rosen20.hdf 5

Screenshots of the output are shown in Figure 6. With just 20 points, our response surface approximation is not very accurate; see the actual function in Figure 1.

To get a better response surface, you could just rerun puq with a larger sample number instead of 20. Or if you wanted to keep the existing data and add more samples, you could do so with 'puq extend'

$>$ puq extend --num 480 rosen 20 

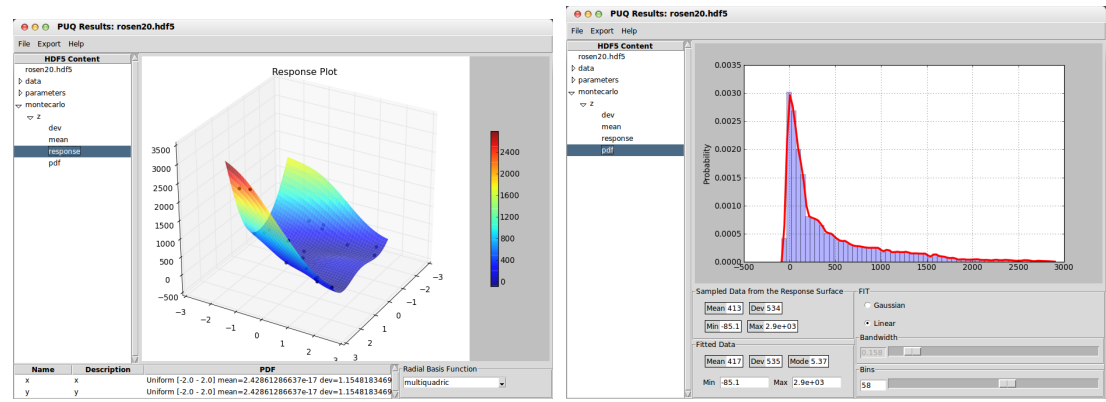

Figure 6: Response Surface and PDF - 20 sample Monte Carlo
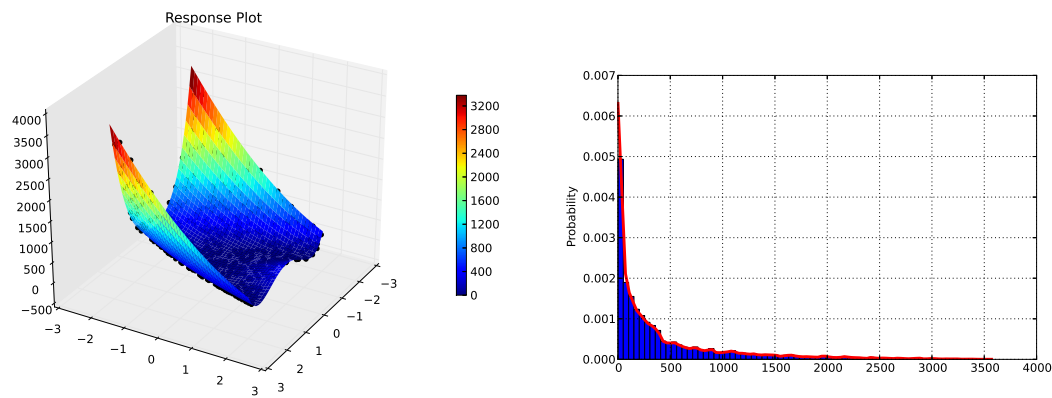

Figure 7: Response Surface and PDF - 500 sample Monte Carlo

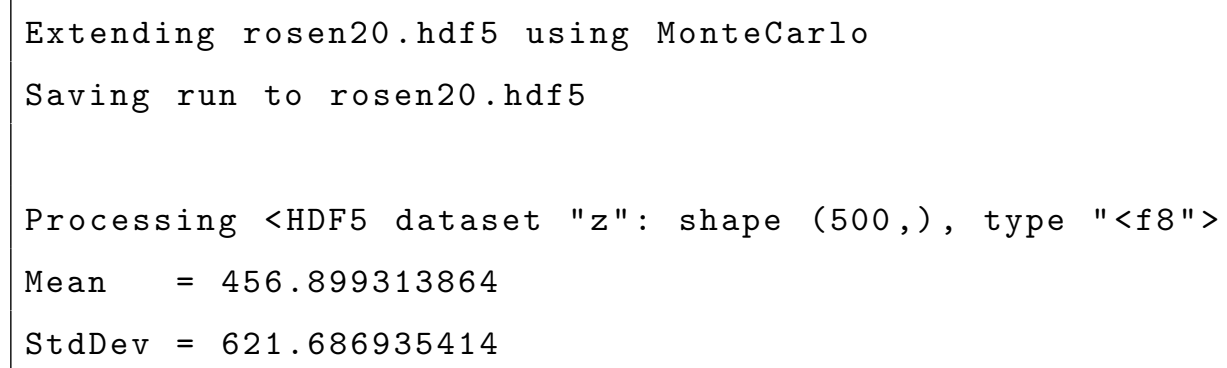

We added 480 more sample points bringing the total to 500 . Now the response surface and PDF (Figure 7) look very much like the real function.

\subsection{Latin hypercube sampling}

PUQ also implements LHS sampling. LHS works similarly to Monte Carlo sampling, but it typically converges to a response surface and mean with less 
samples than Monte Carlo. The control script needs only a single line change from Monte Carlo example.

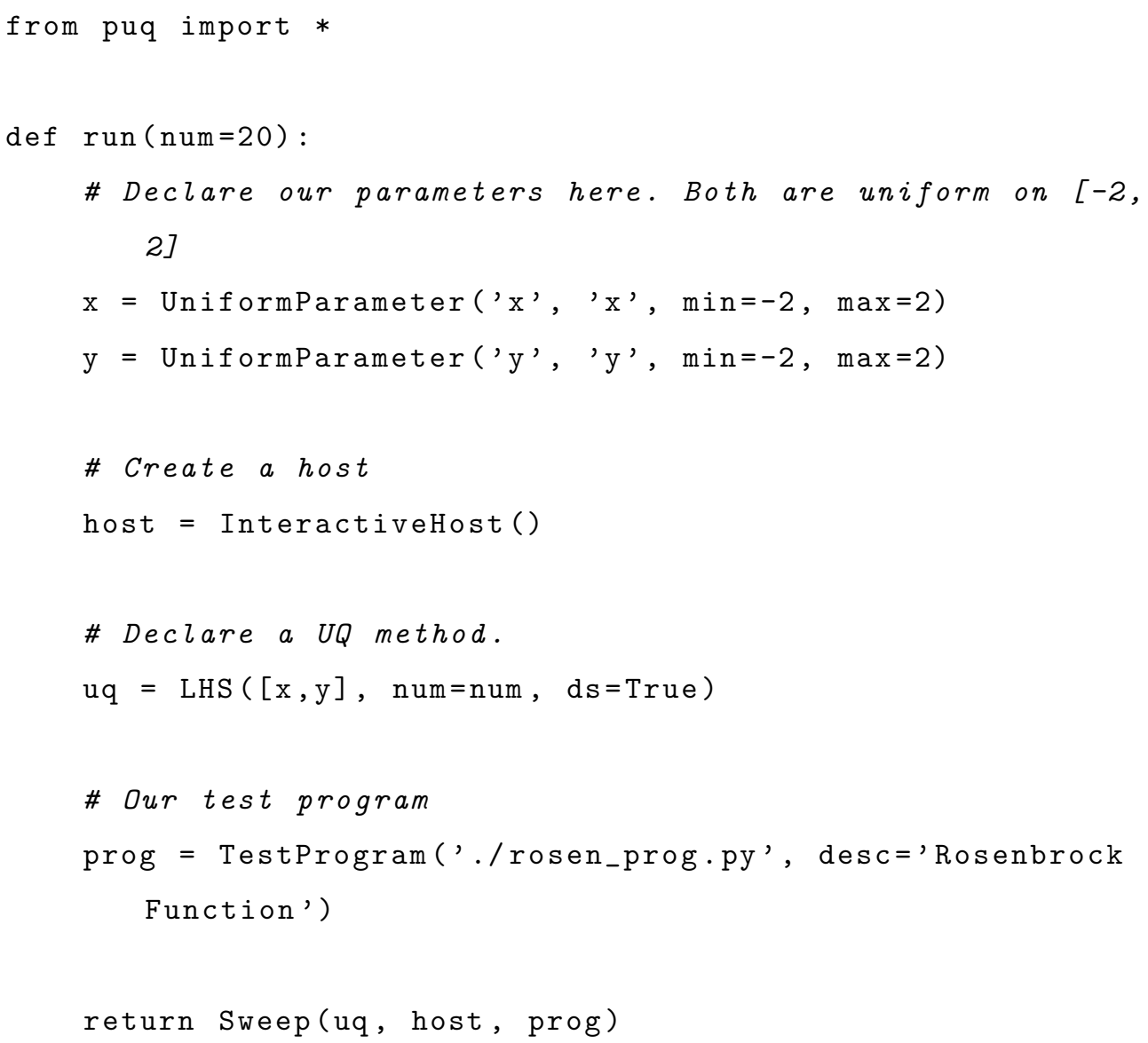

The output appears in Figure 8 

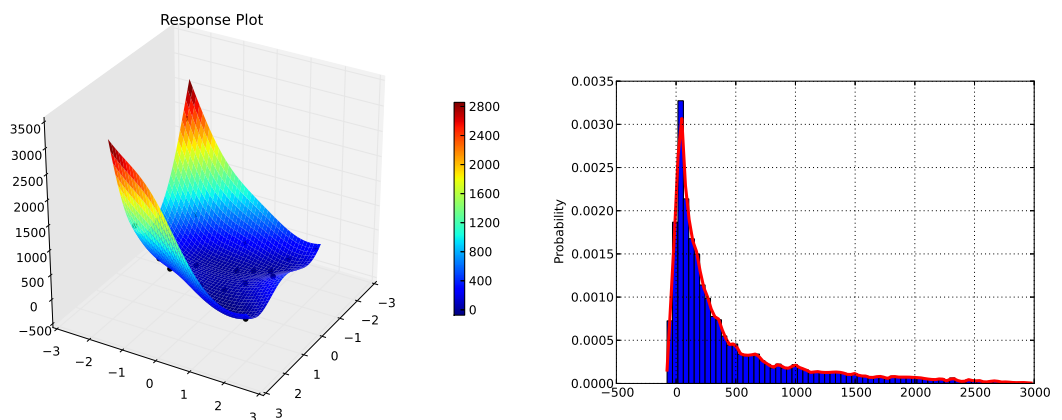

Figure 8: Response Surface and PDF - 20 sample Latin hypercube

\subsection{Smolyak sparse grids and generalized polynomial chaos}

As discussed above gPC [16] is a widely used method that usually exhibits much faster convergence than MC and LHC. It is most suitable for smooth response functions that can be represented as low-order polynomials.

PUQ implements gPC using Legendre polynomials; to compute surrogate models all input parameters are considered as uniformly distributed over their range. For input variables with long-tailed distributions, they are trimmed to $99.9 \%$. After building a surrogate using gPC UP is performed by sampling the input parameters with their actual distributions using LHS. The response function is then called with those samples to build an output PDF.

Response functions and output PDFs can be saved to databases, allowing reuse later. For example an output PDF may be used as the input for another simulation.

Sample points for the input parameters are chosen using a multidimensional sparse grid based on the Clenshaw-Curtis formula. The user must specify a level for the grid which corresponds to the polynomial degree of the response function the gPC code will create. For example, a grid of level 
of 3 will contain the points necessary to perfectly fit a 3rd order polynomial response function.

For our example using the Rosenbrock function, we can use gPC by simply changing the UQ method to Smolyak.

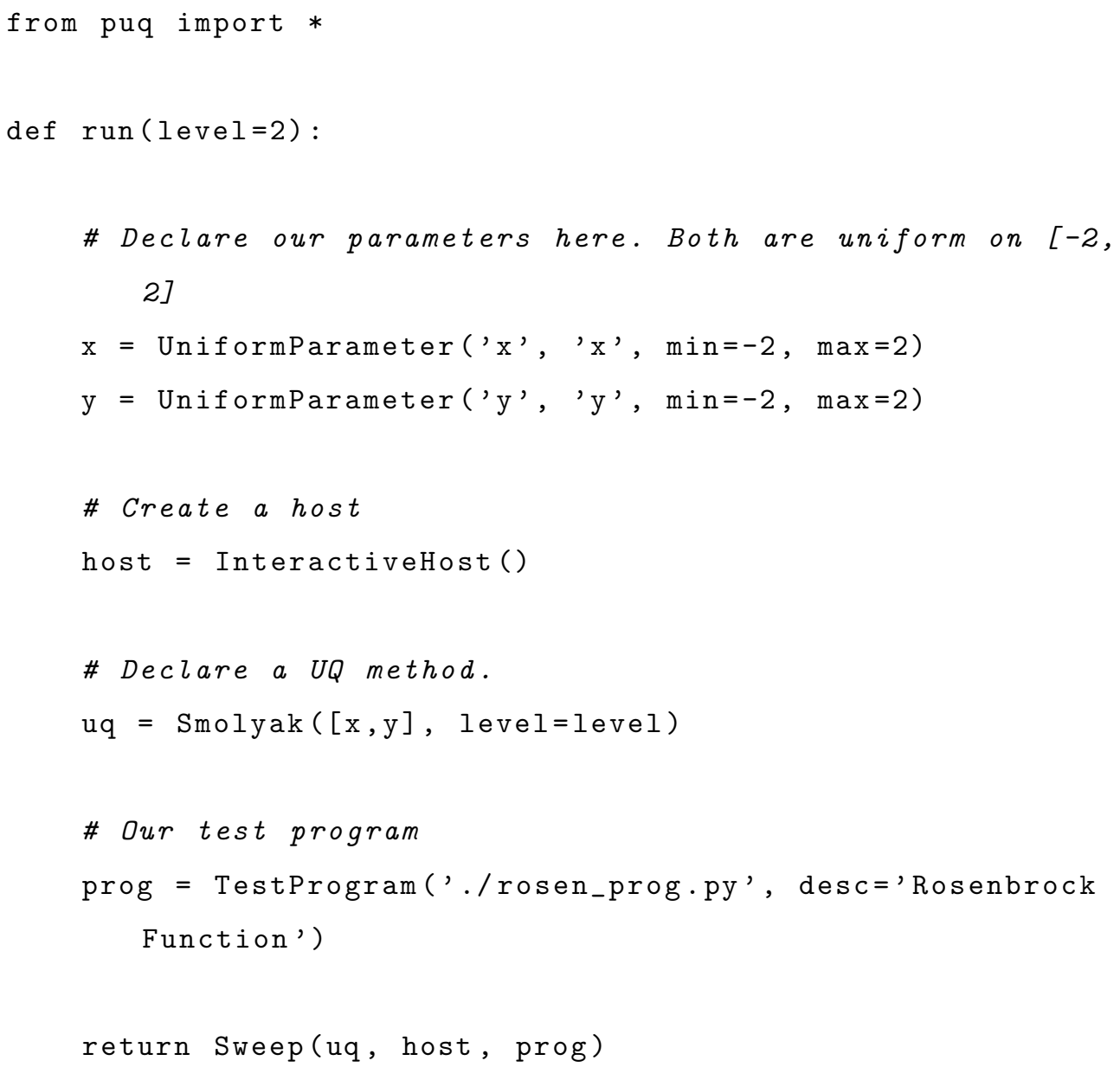




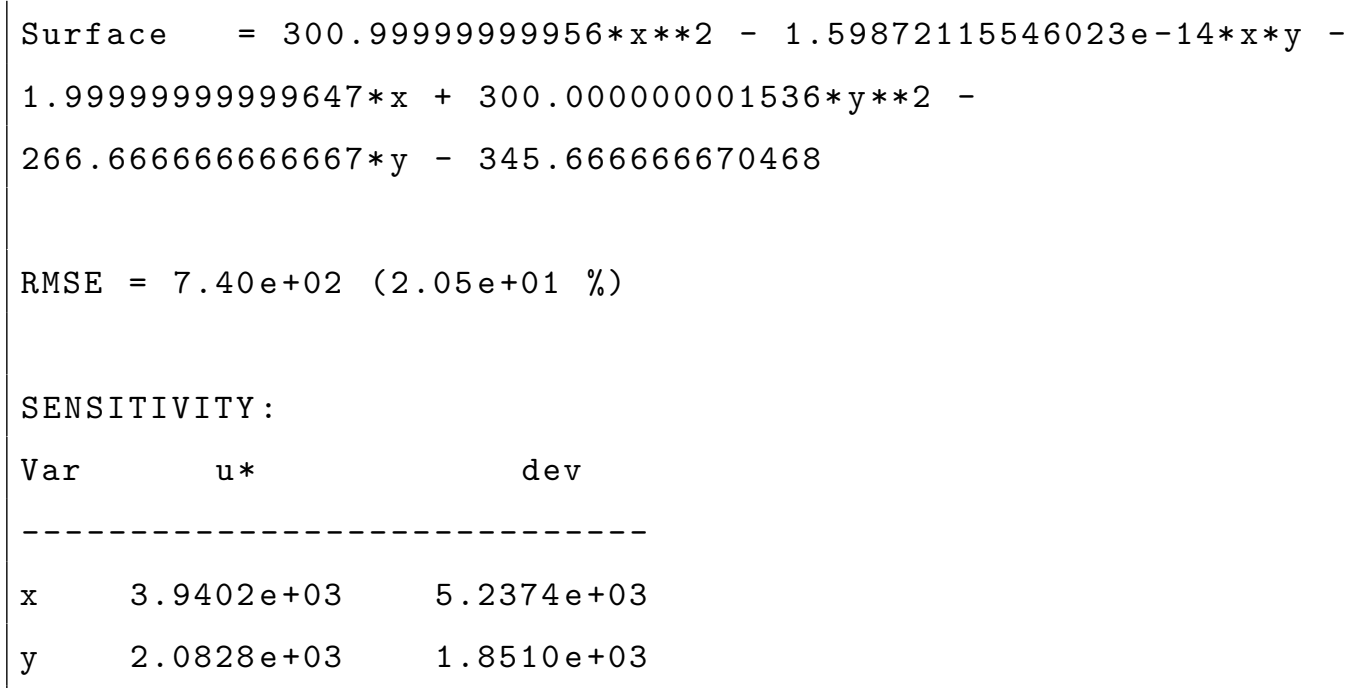

Using 13 points in a level 2 sparse grid, PUQ builds a response surface and prints it as a polynomial. It also calculates the response surface error as $20.5 \%$. This indicates the response surface does not accurately fit the sample points. A higher-order polynomial is needed. Fortunately this is easy to do with puq extend. Because higher-level grids contain all the points in lower-order grids, PUQ can reuse all the previous work and only run the jobs necessary to add the additional points in the new higher-order grid.

See Figure 10 for the level 2 response surface. As indicated by the high error, it is not a good match for the Rosenbrock function. We can get a better match by using a higher-order polynomial:

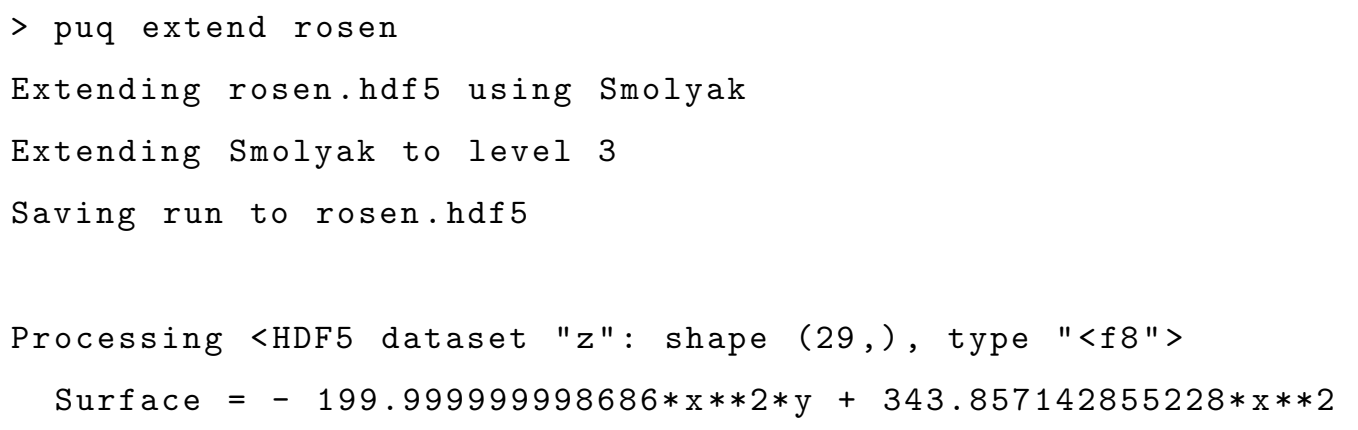




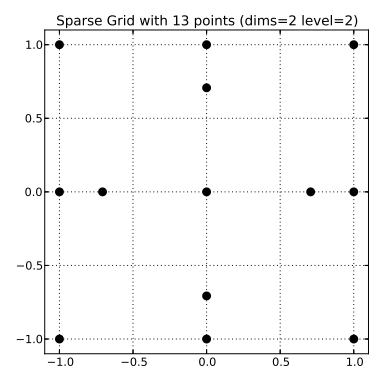

(a) 2 dimension level 2 grid

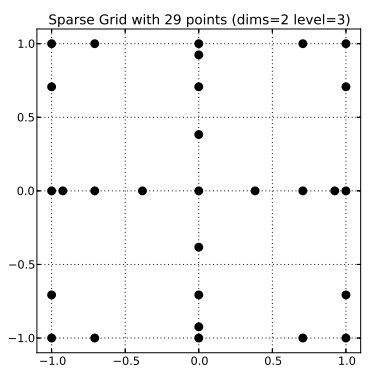

(b) 2 dimension level 3 grid

Figure 9: 2D Clenshaw-Curtis Sparse grids

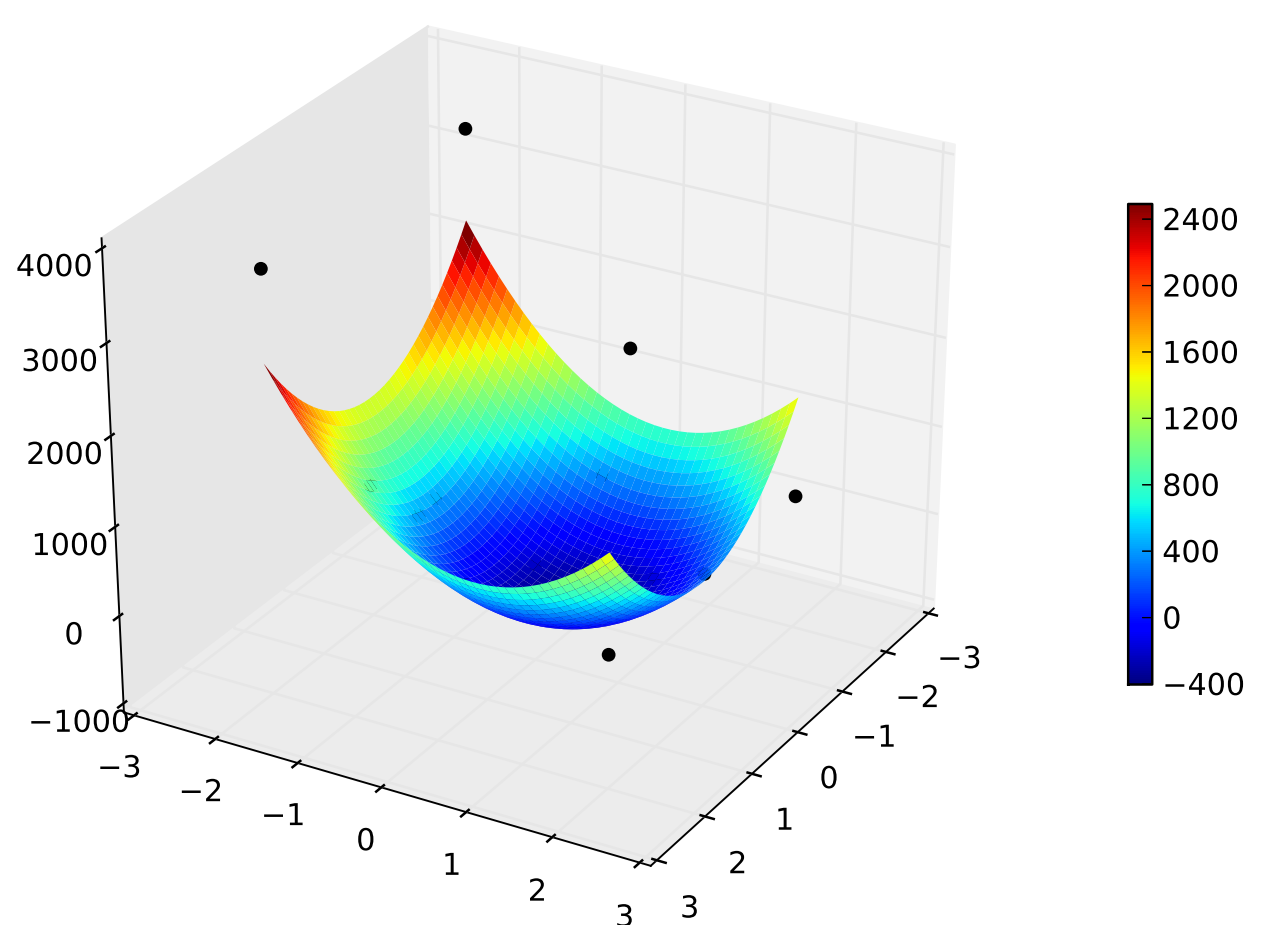

Figure 10: Response Surface from level 2 gPC 


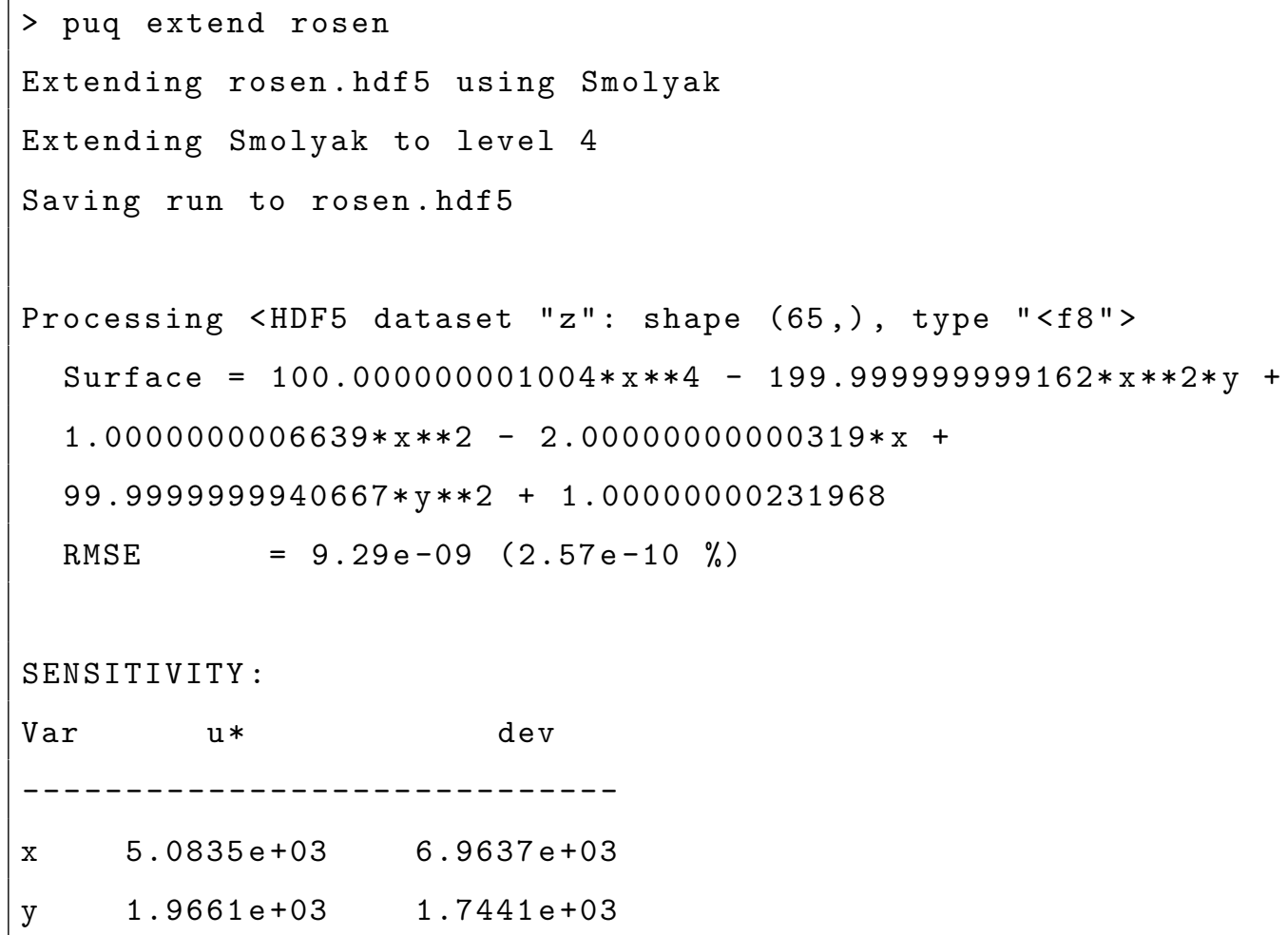




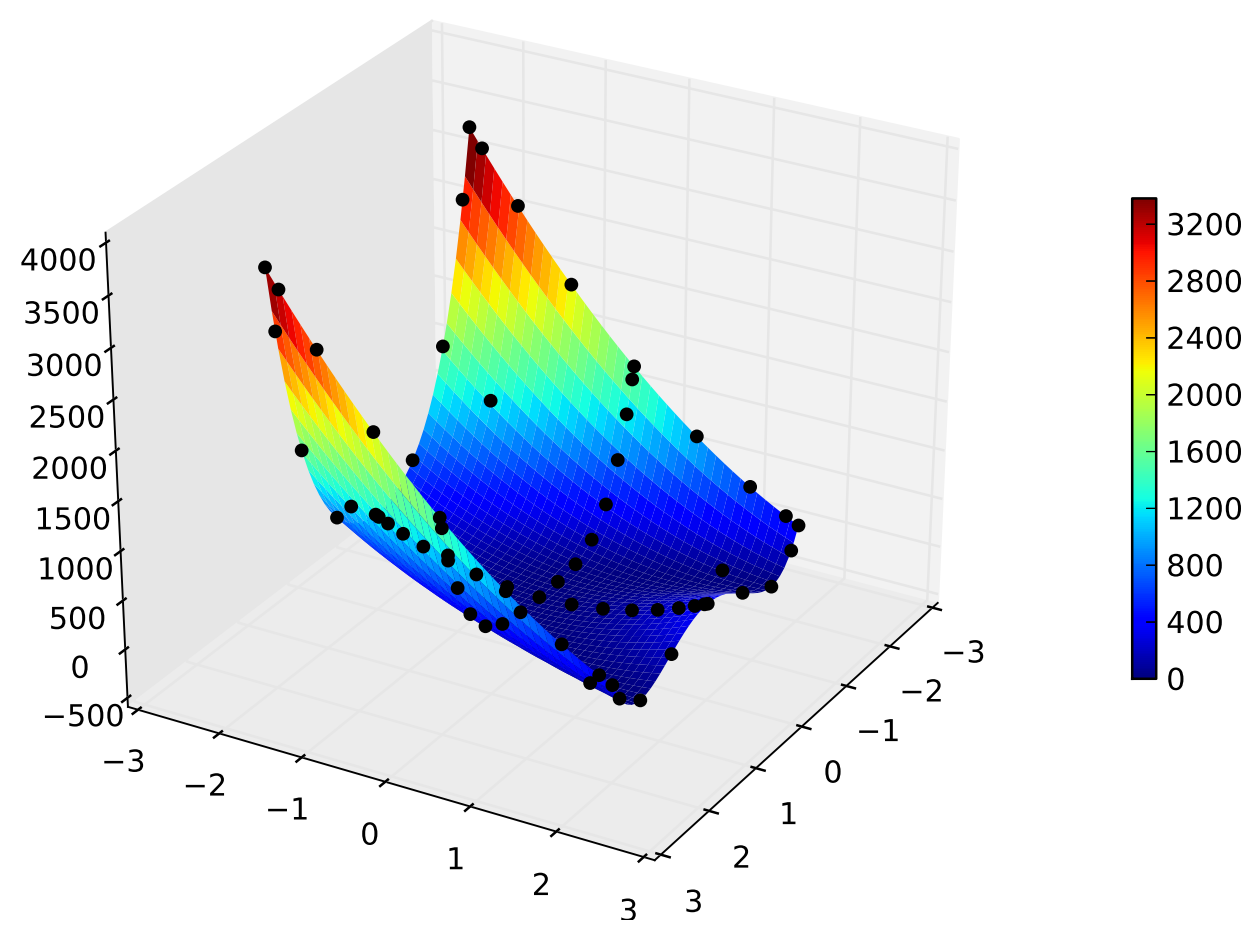

Figure 11: Response Surface from level $4 \mathrm{gPC}$

With a level 4 Smolyak (gPC) run, the response surface is the Rosenbrock function with an error close to zero. This is exactly what we expected because the Rosenbrock function is a 4th-order polynomial. With only 65 samples we have an excellent response surface plus sensitivity analysis.

The Smolyak gPC method in PUQ is recommended because of its quick convergence, built-in error measurement and sensitivity analysis. If the response surface is too rough or has discontinuities, the error may not decrease significantly or may increase due to overfitting as levels increase. In this case, you may prefer to use LHS. A new version of PUQ under active development contains more more advanced methods, such as Gaussian Process Regression, 
which should result in better response surfaces and better understanding of the errors in the response surface.

\subsection{Job submission and parallelism}

UQ is normally an embarrassingly parallel problem; requiring many jobs differing only in the values of some input parameters. Such workloads are a perfect match for both supercomputer clusters and distributed platforms such as BOINC [17].

PUQ currently supports parallel execution on the local host as well as different versions of the Portable Batch System (PBS) on clusters. On the HUBzero 18 and nanoHUB platforms, PUQ uses the submit[19] command which makes use of the Pegasus Workflow Management System[20]. BOINC support is under development.

\subsection{PUQ on HUBzero and nanoHUB}

One of our major goals for PUQ is its integrating it into nanoHUB and HUBzero platform. nanoHUB [5, 6] is a cyber-infrastructure project that enables cloud scientific computing; users can run fully interactive simulations using a standard web-browser or iPad app without the need to download and install any software. HUBzero[18] is the platform that powers nanoHUB and many other HUBs. The nanoPLASTICITY tool is a first step towards the goal of having automated UP for all tools in nanoHUB.

\section{5. nanoPLASTICITY UQ tool in nanoHUB}

Plastic deformation in polycrystalline materials is governed by the nucleation and motion of dislocations inside grains and by grain boundaries 
(GB) slide. The evolution of intra-granular dislocations depends on grain size as grain boundaries act as dislocation sources but also represent barriers to their glide, impeding their motion. The latter results in an increase in the yield stress as grain size is reduced known as Hall-Petch effect [21, 22]. On the other hand, reducing grain size increases the volume fraction of GB and GB deformation mechanisms become more important. Therefore, when the grain size is reduced below a threshold the yield stress decreases, this is known as the inverse Hall-Petch effect [23, 24] Experiments and simulations show that the transition from Hall-Petch to inverse Hall-Petch depends not only on the grain size but also on the properties of the grain boundaries. The nanoPLASTICITY tool [4] was designed to investigate the competing grain-boundary and intragranular dislocation-mediated deformation mechanisms in nanocrystalline metals. The model is based on a multi phase field model in which dislocation and grain boundary sliding are represented by means of phase fields developed by Koslowski et al. [25].

The simulations are carried out in an arrangement of grains with size ranging from 8 to 128 Burgers vectors and periodic boundary conditions. As a simplification, we assume that all the grains have the same crystallographic orientation. Figure 12 shows a schematic representation of the set up and the [111] direction normal to the dislocation slip planes. Grain boundaries are assumed to be planar and we consider grain boundary sliding tangential to the grain boundary surface. Under an externally applied stress the sample deforms plastically by dislocation nucleation and glide and by grain boundary slide. Step-by-step instructions on how to perform the simulations described below can be found in Ref. [26]. 


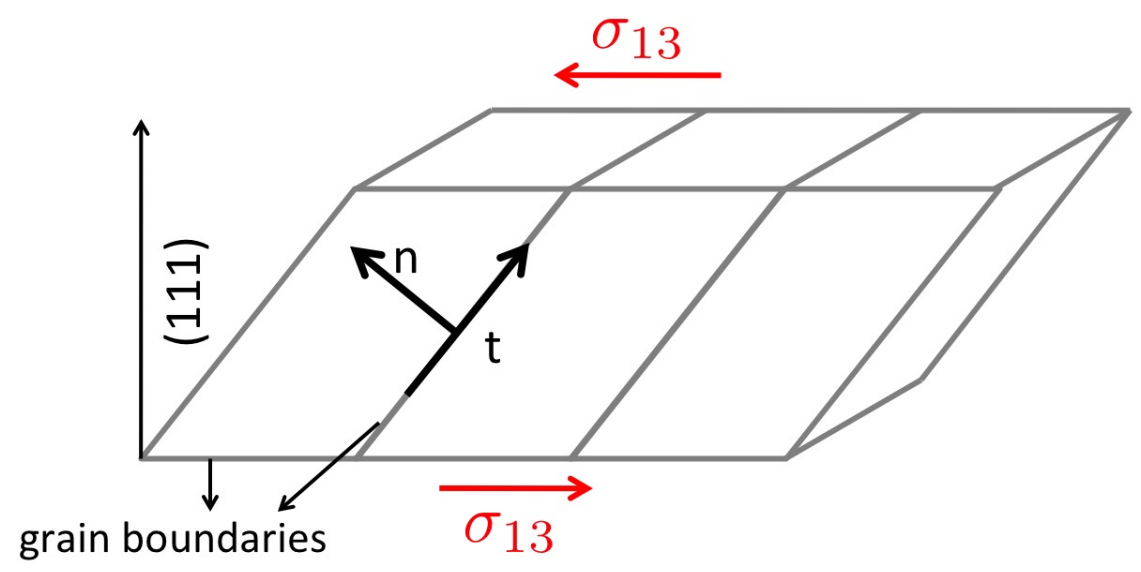

Figure 12: Polycrystalline sample simulated in the nanoPLASTICITY 


\subsection{Phase field approach to plasticity}

In phase field dislocation models, dislocations are described in terms of phase fields, $\xi(x)$, that account for the amount of slip in each crystallographic slip system resolving length and time scales such that individual dislocations can be followed during the simulations. Similarly, the amount of slip due to grain boundary sliding among grains is tracked using a separate phase field, $\phi(x)$.The plastic strain can be written as the sum of the phase fields as:

$$
\varepsilon_{i j}^{p}=\frac{b}{2}\left(\sum_{\alpha=1}^{N} \xi^{\alpha}(x) m_{i}^{\alpha} s_{j}^{\alpha} \delta_{s_{\alpha}}+\sum_{\beta=1}^{M} \phi^{\beta}(x) n_{i}^{\beta} t_{j}^{\beta} \delta_{s_{\beta}}\right)
$$

where the first sum is over all the slip planes and the second is over the grain boundaries. In equation (7), $m$ is the normal to the slip plane, $s$ is the Burgers vector direction, $b$ is the magnitude of the Burgers vector, $n$ is normal and $t$ is tangential to the grain boundary. $\delta_{s_{\alpha}}$ and $\delta_{s_{\beta}}$ are Dirac deltas supported on the slip planes and grain boundaries.

The evolution of the phase fields follows from the minimization of the energy of the system. The energy terms include the strain energy, $E^{\text {int }}$; the external energy due to an externally applied stress, $E^{e x t}$; the Peierls energy of the dislocations, $E^{P}$; and the sliding energy of the grain boundaries, $E^{C}$. Therefore, the equilibrium of the system is found by solving the following system of coupled equations:

$$
\begin{aligned}
& \frac{\delta\left(E^{i n t}[\bar{\xi}, \bar{\phi}]+E^{e x t}[\bar{\xi}, \bar{\phi}]+E^{P}[\bar{\xi}]\right)}{\delta \xi^{\alpha}}=0 \\
& \frac{\delta\left(E^{i n t}[\bar{\xi}, \bar{\phi}]+E^{e x t}[\bar{\xi}, \bar{\phi}]+E^{C}[\bar{\phi}]\right)}{\delta \phi^{\beta}}=0
\end{aligned}
$$


where $\bar{\xi}$ and $\bar{\phi}$ represent an array with the phase fields. The terms in the Eq. 8 and Eq. 9 are explained in detail in [25] [27]. The first is the strain energy and has the form

$$
E^{i n t}=\int_{B} c_{i j k l}\left(\varepsilon_{i j}-\varepsilon_{i j}^{p}\right)\left(\varepsilon_{k l}-\varepsilon_{k l}^{p}\right) d^{3} x
$$

where $\varepsilon_{i j}$ is the strain tensor and the isotropic tensor of elastic constants is:

$$
c_{i j k l}=\frac{E_{\nu}}{(1+\nu)(1-2 \nu)} \delta_{i j} \delta_{k l}+\frac{E}{2(1+\nu)}\left(\delta_{i k} \delta_{j l}+\delta_{i l} \delta_{j k}\right)
$$

The Youngs modulus, E, and the Poisson ratio, $\nu$, can be entered in the nanoPLASTICITY tool for different materials. The external energy is:

$$
E^{e x t}=\int_{B} \sigma_{i j}^{e x t} \varepsilon_{i j} d^{3} x
$$

The Peierls stress takes into account the periodicity of the lattice and it is represented by a sinusoidal functional:

$$
E^{P}=\sum_{\alpha} \int_{S} P \sin ^{2}\left(2 \pi \xi^{\alpha}\right) d^{2} x
$$

where the integrals are in the slip planes and the cohesive energy of the grain boundary is also approximated similarly with integrals over the surface of the grain boundaries:

$$
E^{C}=\sum_{\beta} \int_{S_{G}} G \sin ^{2}\left(2 \pi \phi^{\beta}\right) d^{2} x
$$

The coefficients $P$ and $G$ determine the resistance to dislocation glide and to grain boundary sliding and their values can be entered in the nanoPLASTICITY tool. Their magnitudes can be calculated from molecular dynamics simulations or determined experimentally [28]. 
The nanoPLASTICITY tool launches simulations that calculate the evolution of dislocations to predict the elastic and plastic response of a polycrystalline sample. Figure 13 shows the von Mises stress versus the von Mises strain of a polycrystalline sample with grain size $d=32 \mathrm{~b}$. The yield stress is calculated using the $0.2 \%$ offset as shown in Figure 13

\subsection{PUQ driving plasticity simulations}

All input parameters in the nanoPLASTICITY tool can be specified in terms of a single value or a distribution (normal or uniform). These inputs include the Burgers vector, elastic constants (Young's modulus E and Poisson's ratio $\nu$ ) as well as the activation energies for dislocation glide (Peierls energy) and grain boundary slide, $P$ and $G$ respectively. Figure 14 and 15 show the PDF of the yield stress calculated with the input parameters in in Table 1 with sample sizes ranging from $\mathrm{d}=16 \mathrm{~b}$ to $128 \mathrm{~b}$ and two different distributions of GB energy $G$; the figures also show the sensitivity of the yield stress with respect to the input parameters.

Figure 13 shows the yield stress as a function of the grain size calculated with the nanoPLASTICITY tool with the coefficients in Table1, The Peierls energy is fixed to $0.6 \mathrm{~J} / \mathrm{m}^{2}$ in all simulations, while the GB is varied from $0.05 \mathrm{~J} / \mathrm{m}^{2}$ to $0.05 \mathrm{~J} / \mathrm{m}^{2}$. As expected, when the GB sliding energy is high, $G=1.0 \mathrm{~J} / \mathrm{m}^{2}$, the yield stress increases with decreasing grain size following the Hall-Petch relation. Due to the high value of the GB energy [25], the only active deformation mechanism is dislocation motion and multiplication. This renders a higher yield stress for smaller grain size where dislocation activity is limited. When the mean GB sliding energy is reduced below $G=0.15 \mathrm{~J} / \mathrm{m}^{2}$, GB sliding becomes active and the inverse Hall-Petch effect is observed. It is 
important to notice that the value maximum yield stress and the grain size at which it occurs depends on the GB energy. The maximum yield stress is observed at $8 \mathrm{~nm}$ grain size for $G=0.15 \mathrm{~J} / \mathrm{m}^{2}$ and $G=0.20 \mathrm{~J} / \mathrm{m}^{2}$ and at 16 $\mathrm{nm}$ for $G=0.10 J / \mathrm{m}^{2}$. No maximum is observed in the range $4 \mathrm{~nm}$ to $64 \mathrm{~nm}$ for $G=0.05 \mathrm{~J} / \mathrm{m}^{2}$.

The error bars are calculated from the full-width at half-maximum of the pdfs shown in Figures 14 and 15 . These figures also show the sensitivity coefficients of the GB sliding energy and the Peierls energy. When the GB sliding energy is high, plastic deformation proceeds from dislocation nucleation and glide only; therefore, the yield stress is not affected by the GB sliding energy. The results also show that smaller grain sizes are more sensitive to variations of the Peierls energy. The sensitivity to the GB energy is 3 to 5 orders of 


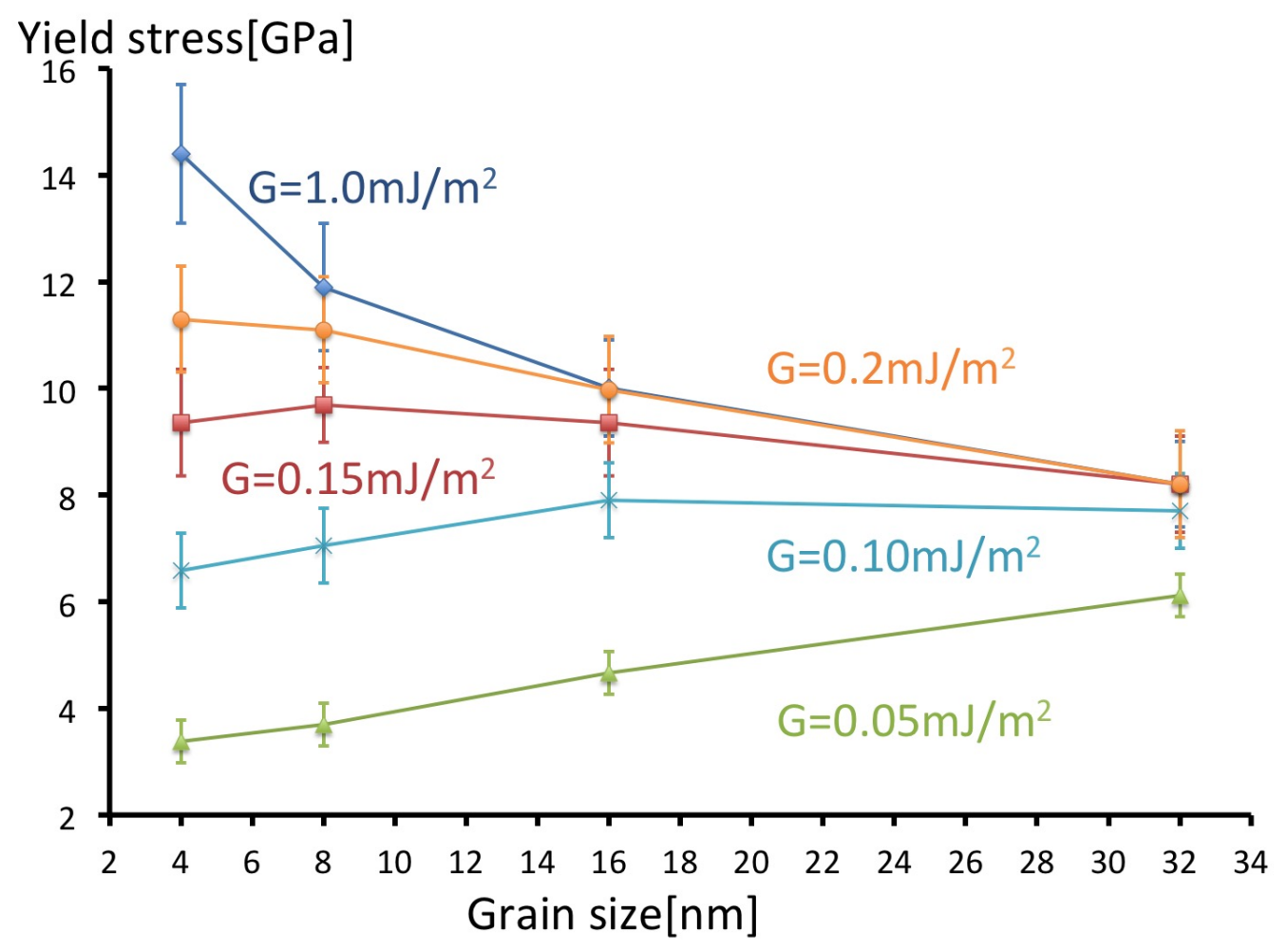

Figure 13: Yield stress vs grain size calculated with the nanoPLASTICITY tool with the coefficients in Table 1 
magnitude smaller and not visible in Figure 14.

Figure 15 shows the pdf of the yield stress and the sensitivity coefficients calculated with the parameters in Table 1 and $G=0.15 \mathrm{~J} / \mathrm{m}^{2}$. The samples with smaller grain size and therefore, greater volume fraction of grain boundaries have a larger sensitivity coefficient for the GB sliding energy.

Figure 16 shows first, second, and their order gPC surrogate models of the yield stress with Young's moduli with a mean of $200 \mathrm{GPa}$ and standard deviation of $10 \mathrm{GPa}$; Peierls stress with mean $0.6 \mathrm{~J} / \mathrm{m}^{2}$ and standard deviation of $0.06 \mathrm{~J} / \mathrm{m}^{2}$ and GB glide activation energy of $0.15 \mathrm{~J} / \mathrm{m}^{2}$ with standard deviation of $0.01 \mathrm{~J} / \mathrm{m}^{2}$. First order response surfaces only show the relative sensitivity of each variable, yield stress is more sensitive to Peierls energy than to Young's modulus and Peierls energy and GB glide energy have approximately equal importance. The second order response surfaces provide additional insight. Increasing the Peierls energy that controls dislocation glide tends to increase the yield stress but only up to a threshold when grain boundary glide takes over as a dominant deformation mechanism. This threshold increases with increasing GB glide energy. The RMS error of the surrogate model decreases going from 1st order to 2nd indicating that higher order is needed. This is not the case going to 3rd order where the RMS error increases and the response surfaces show over-fitting. The polynomial order required for an appropriate description will depend on the actual underlying model and the range of parameters explored; nanoPLASTICITY and PUQ users should perform tests such as the ones shown here to establish an appropriate response surface for their problem. 

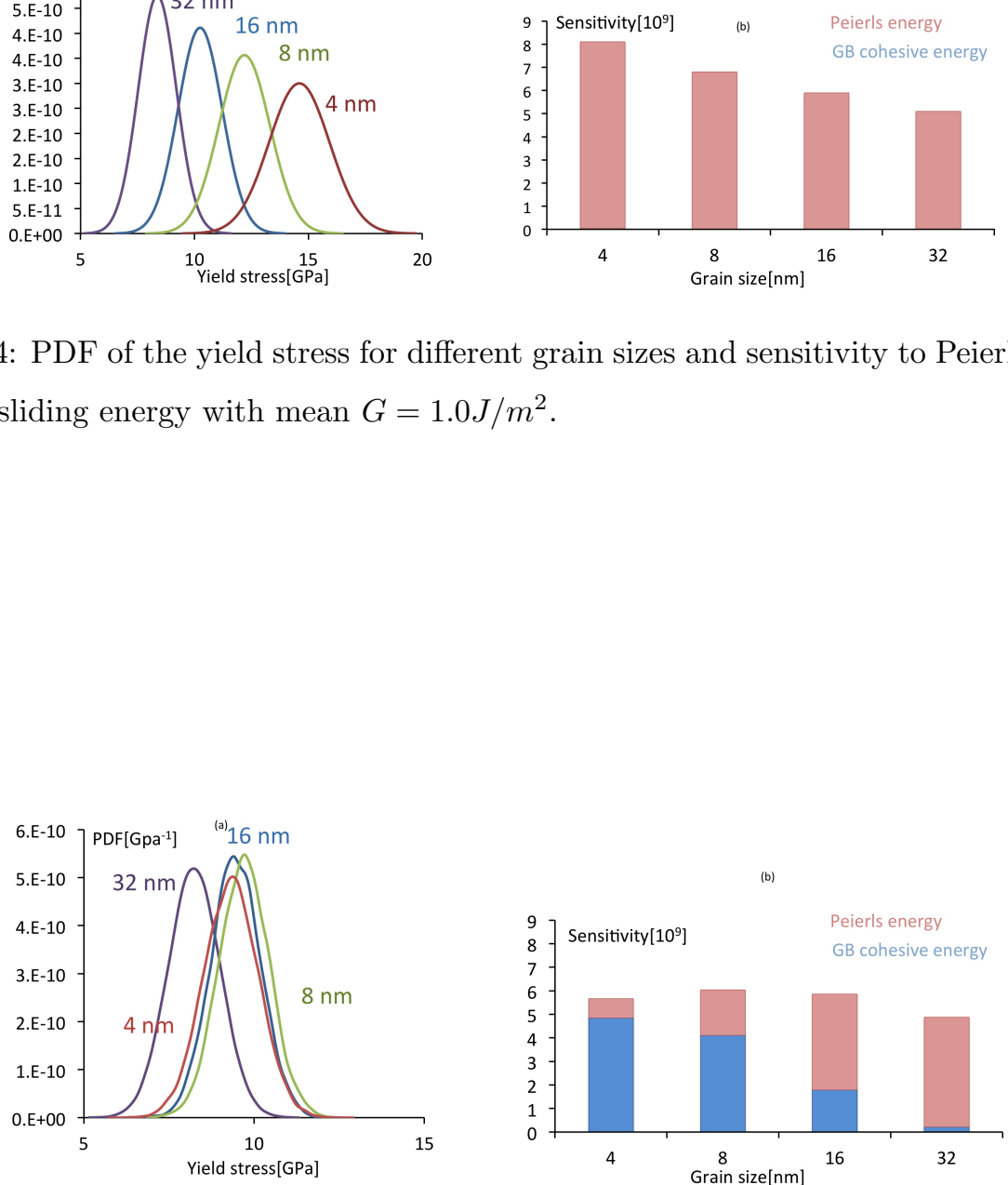

Figure 14: PDF of the yield stress for different grain sizes and sensitivity to Peierls energy and GB sliding energy with mean $G=1.0 \mathrm{~J} / \mathrm{m}^{2}$.

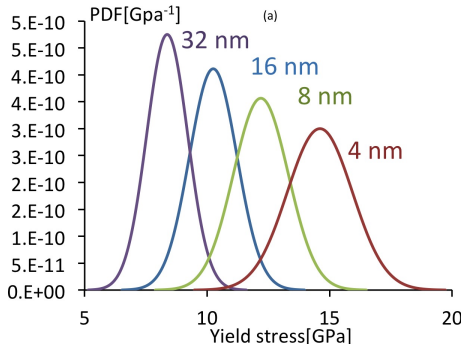

Figure 15: PDF of the yield stress for different grain sizes and sensitivity to Peierls energy and GB sliding energy with mean $G=0.15 \mathrm{~J} / \mathrm{m}^{2}$. 


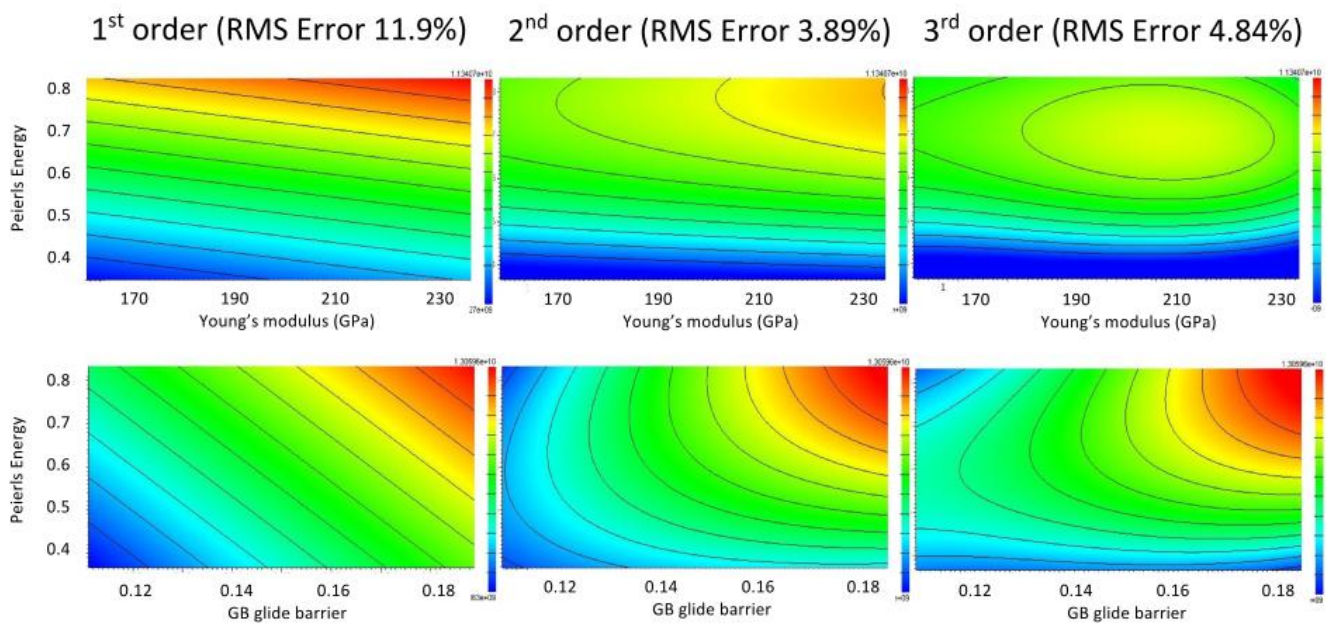

Figure 16: Response surface functions of the yield surface.

\section{Conclusions}

In summary, we introduce the PUQ tool for uncertainty propagation and surrogate model construction and sensitivity analysis. PUQ is easy to use, light-weight and non-intrusive requiring only tagging of the QoI in the simulation code. PUQ can be downloaded or used directly in a nanoHUB workspace. It also orchestrates simulations in the nanoPLASTICITY tool that enables propagation of uncertainties in a dislocation dynamics code. 


\section{Acknowledgements}

This work was partially supported by Purdue's Center for the Prediction of Reliability, Integrity, and Survivability of Microsystems funded by the U.S. Department of Energy National Nuclear Security Administration under Contract No. DE-FC52-08NA28617 and by the Network for Computational Nanotechnology funded by the US National Science Foundation under grant EEC-0228390.

\section{References}

[1] Scientific grand challenges in national security: The role of computing at the extreme scale.

URL http://science.energy.gov/ /media/ascr/pdf/ program-documents/docs/Nnsa_grand_challenges_report.pdf

[2] J. Allison, L. M, W. C, S. X, Virtual aluminum castings: an industrial application of ICME, JOM 58 (2006) 28-35.

[3] J. C. Helton, Conceptual and computational basis for the quantification of margins and uncertainty, Tech. rep., Sandia National Laboratories (United States). Funding organisation: US Department of Energy (United States) (2009).

[4] M. Hunt, L. Cao, A. Strachan, M. Koslowski, Nanoplasticity lab (Feb 2014). doi:doi:10.4231/D3D21RK33.

URL https://nanohub.org/resources/22119 
[5] S. A, K. G, L. M, Cyber-Enabled Simulations in Nanoscale Science and Engineering INTRODUCTION, Computing In Science \& Engineering 12 (2010) 12-17.

[6] K. Madhavan, L. Zentner, V. Farnsworth, S. Shivarajapura, M. Zentner, N. Denny, G. Klimeck, Nanohub. org: Cloud-based services for nanoscale modeling, simulation, and education, Nanotechnology Reviews 2 .

URL http://www.degruyter.com/view/j/ntrev.2013.2.issue-1/ ntrev-2012-0043/ntrev-2012-0043.xml

[7] A. Strachan, S. Mahadevan, V. Hombal, L. Sun, Functional derivatives for uncertainty quantification and error estimation and reduction via optimal high-fidelity simulations, MODELLING AND SIMULATION IN MATERIALS SCIENCE AND ENGINEERING 21 (6). doi:\{10. 1088/0965-0393/21/6/065009\}.

[8] D. W. Scott, Multivariate density estimation: theory, practice, and visualization, Vol. 383, John Wiley \& Sons, 2009.

[9] M. McKay, R. Beckman, W. Conover, A comparison of three methods for selecting values of input variables in the analysis of output from a computer code, Technometrics 42 (1) (2000) 55-61.

[10] D. Xiu, G. Karniadakis, The Wiener-Askey polynomial chaos for stochastic differential equations, SIAM JOURNAL ON SCIENTIFIC COMPUTING 24 (2) (2002) 619-644. doi:\{10.1137/ S1064827501387826\}. 
[11] N. Wiener, The homogeneous chaos, AMERICAN JOURNAL OF MATHEMATICS 60 (1938) 897-936. doi:\{10.2307/2371268\}.

[12] M. Eldred, C. Webster, P. Constantine, Evaluation of non-intrusive approaches for wiener-askey generalized polynomial chaos, 2008.

URL http://dakota.sandia.gov/papers/Poly_Chaos.pdf

[13] E. Jones, T. Oliphant, P. Peterson, et al., Scipy: Open source scientific tools for python (2001).

URL http://docs.scipy.org/doc/scipy-0.14.0/reference/ generated/scipy.interpolate.Rbf.html

[14] M. D. Morris, Factorial sampling plans for preliminary computational experiments, Technometrics 33 (2) (1991) 161-174. arXiv:http:// Www.tandfonline.com/doi/pdf/10.1080/00401706.1991.10484804 doi:10.1080/00401706.1991.10484804.

URL http://www.tandfonline.com/doi/abs/10.1080/00401706. 1991.10484804

[15] F. Campolongo, J. Cariboni, A. Saltelli, An effective screening design for sensitivity analysis of large models, Environ. Model. Softw. 22 (10) (2007) 1509-1518. doi:10.1016/j.envsoft.2006.10.004.

URL http://dx.doi.org/10.1016/j.envsoft.2006.10.004

[16] D. Xiu, Fast numerical methods for stochastic computations: a review, Communications in computational physics 5 (2-4) (2009) 242-272.

URL http://citeseerx.ist.psu.edu/viewdoc/download?doi=10. 1.1.148.5499\&\#38; rep=rep1\&\#38; type=pdf 
[17] D. P. Anderson, BOINC: A System for Public-Resource Computing and Storage, in: Proceedings of the Fifth IEEE/ACM International Workshop on Grid Computing., GRID '04, IEEE Computer Society, Washington, DC, USA, 2004, pp. 4-10. doi:10.1109/grid.2004.14.

URL http://dx.doi.org/10.1109/grid.2004.14

[18] M. McLennan, R. Kennell, Hubzero: A platform for dissemination and collaboration in computational science and engineering., Computing in Science and Engineering 12 (2) (2010) 48-53.

URL http://ieeexplore.ieee.org/xpl/articleDetails.jsp? arnumber $=5432299$

[19] M. McLennan, S. Clark, F. McKenna, E. Deelman, M. Rynge, K. Vahi, D. Kearney, C. Song, Bringing scientific workflow to the masses via pegasus and hubzero., in: T. Kiss (Ed.), IWSG, Vol. 993 of CEUR Workshop Proceedings, CEUR-WS.org, 2013.

URL http://dblp.uni-trier.de/db/conf/iwsg/iwsg2013.html\# McLennanCMDRVKS13

[20] E. Deelman, G. Singh, M.-H. Su, J. Blythe, Y. Gil, C. Kesselman, G. Mehta, K. Vahi, G. B. Berriman, J. Good, A. Laity, J. C. Jacob, D. S. Katz, Pegasus: A framework for mapping complex scientific workflows onto distributed systems, Sci. Program. 13 (3) (2005) 219-237. URL http://dl.acm.org/citation.cfm?id=1239649.1239653

[21] W. Sylwestrowicz, E. Hall, The deformation and ageing of mild steel, Proceedings of the Physical Society. Section B 64 (6) (1951) 495. 
[22] N. J. Petch, Cleavage strength of polycrystals, J. Iron Steel Inst. 174 (Part 1) (1953) 25-28.

[23] J. Schiøtz, K. W. Jacobsen, A maximum in the strength of nanocrystalline copper, Science 301 (5638) (2003) 1357-1359. arXiv: http://www. sciencemag.org/content/301/5638/1357.full.pdf, doi:10.1126/science.1086636. URL http://www.sciencemag.org/content/301/5638/1357. abstract

[24] M. Meyers, A. Mishra, D. Benson, Mechanical properties of nanocrystalline materials, Progress in Materials Science 51 (4) (2006) 427 - 556. doi:DOI:10.1016/j.pmatsci.2005.08.003.

URL http://www.sciencedirect.com/science/article/pii/ S0079642505000447

[25] M. Koslowski, D. W. Lee, L. Lei, Role of grain boundary energetics on the maximum strength of nanocrystalline nickel, Journal of the Mechanics and Physics of Solids 59 (7) (2011) 1427-1436.

[26] M. Hunt, M. Koslowski, A. Strachan, Reproducing results from "puq: a code for non-intrusive uncertainty propagation in computer simulations" (Mar 2015).

URL https://nanohub.org/resources/22119

[27] A. Hunter, F. Saied, C. Le, M. Koslowski, Large-scale 3d phase field dislocation dynamics simulations on high-performance architectures, Int. J. High Perform. Comput. Appl. 25 (2) (2011) 223-235. doi: 
URL http://dx.doi.org/10.1177/1094342010382534

[28] D. W. Lee, H. Kim, A. Strachan, M. Koslowski, Effect of core energy on mobility in a continuum dislocation model, Physical Review B 83 (10) (2011) 104101. 